\title{
NEW ASYMPTOTIC EXPANSION AND ERROR BOUND FOR STIRLING FORMULA OF RECIPROCAL GAMMA FUNCTION
}

\author{
PEDRo J. PAgOla
}

Abstract. Studying the problem about if certain probability measures are determinate by its moments $[4,8,10]$ is useful to know the asymptotic behavior of the probability densities for large values of argument. This requires, previously, the knowledge of the asymptotic expansion of reciprocal Gamma function $1 / \Gamma(z)$ when $\Re z$ is large and $\mathfrak{I} z$ is fixed [8]. Then, the well known Stirling formula for large $|z|$ of the Gamma function $\Gamma(z)$ or its reciprocal $1 / \Gamma(z)$ is not appropriate for this problem. So, the main aim of this paper is to obtain a new asymptotic expansion for reciprocal Gamma function valid for large $\mathfrak{R} z$ and establish a new explicit error bound for the first term of this expansion, that is, the Stirling formula.

Mathematics subject classification (2010): 33B15, 41A60.

Keywords and phrases: Reciprocal gamma function, asymptotic expansions, error bounds.

\section{REFERENCES}

[1] R. A. Askey, R. Roy, NIST Handbook of Mathematical Functions: Chapter 5, Gamma Function, NIST and Cambridge Univ. Press, New York, 2010.

[2] M. V. Berry And C. J. Howls, Hyperasymptotics for integrals with saddles, Proc. Roy. Soc. London 434, (1991), 657-675.

[3] W. G. C. BoyD, Gamma function asymptotics by an extension of the method of steepest descents, Proc. Roy. Soc. London 447, (1994), 609-630.

[4] P. HÖRFELT, The moment problem for some wiener functionals:corrections to previous proofs (with an appendix by H. L. Pedersen), J. Appl. Prob. 42, (2005), 851-860.

[5] A. Q. LIU, G. F. Li, B. N. GUO, F. QI, Monotonicity and logarithmic concavity of two functions involving exponential function, Internat. J. Math. Ed. Sci. Tech. 39, 5 (2008), 686-691

[6] J. L. LóPEZ, P. J. PAGola And E. PÉRez Sinusía, A systematization of the saddle point method. Application to the Airy and Hankel functions, J. Math. Anal. Appl. 354, 1 (2009), 347-359.

[7] G. NEMES, Error bounds and exponential improvements for the asymptotic expansions of the Gamma function and its reciprocal, Proc. Roy. Soc. Edinburgh 145, 3 (2015), 571-596.

[8] P. J. PAGOLA, Asymptotic behaviour of the density function of the integral of a geometric Brownian motion, Submitted.

[9] N. M. Temme, Special Functions: an Introduction to the Classical Functions of Mathematical Physics, John Wiley and Sons, New York, 1996.

[10] M. Yor, On some exponential functionals of Brownian motion, Adv. Appl. Prob. 24, (1992), 509-531. 